\title{
Aggregation on Finite Ordinal Scales by Scale Independent Functions
}

\author{
Jean-Luc MARICHAL (jean-luc.marichal [at] uni.lu)* \\ Faculty of Law, Economics, and Finance, University of Luxembourg \\ 162A, avenue de la Faïencerie, L-1511 Luxembourg, G.D. Luxembourg. \\ Radko MESIAR (mesiar[at] leo.svf.stuba.sk) ${ }^{\dagger}$ \\ Department of Mathematics, Faculty of Civil Engineering \\ Slovak Technical University, Radlinskeho 11, SK-81368 Bratislava, Slovakia.
}

(Submitted 27 December 2002; revised 11 November 2004)

\begin{abstract}
We define and investigate the scale independent aggregation functions that are meaningful to aggregate finite ordinal numerical scales. Here scale independence means that the functions always have discrete representatives when the ordinal scales are considered as totally ordered finite sets. We also show that those scale independent functions identify with the so-called order invariant functions, which have been described recently. In particular, this identification allows us to justify the continuity property for certain order invariant functions in a natural way.
\end{abstract}

Keywords: Aggregation functions, finite ordinal scales, order invariant functions, smooth discrete functions, lattice polynomials.

2000 Mathematics Subject Classification: Primary: 91C05, 91E45; Secondary: 06A99, 39A12.

\section{Introduction}

Let $A$ be a finite set of alternatives and consider a real interval $E \subseteq \mathbb{R}$. A scale of measurement is a mapping $h: A \rightarrow E$ that assigns real numbers to elements of $A$ according to some criterion. The scale type of a scale is defined by giving a class of admissible transformations, transformations that lead from one scale to another acceptable version of it. For instance, a scale is called an ordinal scale if the class of admissible transformations consists of the increasing bijections (automorphisms) $\phi: E \rightarrow E$. This means that the scale values are determined only up to order. For a general discussion of the theory of scale type and for the definitions of other scale types, see e.g. [10, 11, 20].

It is a common practice to simplify the definition of an ordinal scale by merely giving the sequence of ordered values of the image $h(A) \subseteq E$

* Partially supported by a grant from the David M. Kennedy Center for International Studies, Brigham Young University.

† Partially supported by grants VEGA 1/0273/03 and APVT-20-023402.

(C) 2006 Kluwer Academic Publishers. Printed in the Netherlands. 
of $A$ under $h$. Being of ordinal nature, this sequence is defined up to order, that is, within an automorphism $\phi: E \rightarrow E$.

EXAMPLE $1.1([6,7])$. Suppose we want to evaluate a commodity, e.g. a car, according to a certain ordinal criterion, e.g. comfort, by means of a 5-value ordinal performance scale in $\mathbb{R}$. We can assign the number 1 to a bad comfort, 2 to an acceptable one, 3 to a good one, 4 to a very good one, and 5 to an excellent one. Clearly, we could just as well use the numbers $4,5,7,8,10$, or the numbers $-3,1.5,14.2,58,263$, or any numbers that preserve the order.

By adopting this approach, it is easy to see that a finite ordinal scale can be defined in two equivalent ways; one is numerical and the other is symbolical.

Numerically, a finite ordinal scale is a finite and strictly increasing sequence of $E$ determined up to order and representing the possible rating benchmarks defined along some ordinal criterion; see e.g. [20]. For example, the sequences

$$
(4,5,7,8,10) \text { and }(-3,1.5,14.2,58,263)
$$

represent two equivalent versions of the evaluation scale defined in Example 1.1.

Symbolically, a finite ordinal scale is a finite chain $(S, \preccurlyeq)$, that is a totally ordered finite set, whose elements are ranked according to some ordinal criterion. For example the scale of evaluation of a commodity by a consumer such as

$$
S=\{B \prec A \prec G \prec V G \prec E\}
$$

is a finite ordinal scale, whose elements might refer to the following linguistic terms: bad, acceptable, good, very good, excellent.

The equivalence between these numerical and symbolical definitions follows immediately from the fact that the total order $\preccurlyeq$ defined on $S$ can always be numerically represented in $E$ by means of an order preserving isomorphism $f: S \rightarrow E$ such that

$$
s_{i} \preccurlyeq s_{j} \quad \Leftrightarrow \quad f\left(s_{i}\right) \leqslant f\left(s_{j}\right) \quad\left(s_{i}, s_{j} \in S\right),
$$

see [10, Chapter 1]. Such an isomorphism is defined up to an automorphism $\phi: E \rightarrow E$; that is, with $f$ all functions $f^{\prime}=\phi \circ f$ (and only these) represent the same order on $S$.

Thus, the elements of a finite set $A$ of alternatives can be ordinally evaluated either by means of a numerical mapping $h: A \rightarrow E$, defined up to an automorphism $\phi: E \rightarrow E$ or, equivalently, by a symbolical 
mapping $h^{\prime}: A \rightarrow S$. When $|S|=|h(A)|$, both mappings $h$ and $h^{\prime}$ are connected to each other through the identity $h=f \circ h^{\prime}$, where $f: S \rightarrow E$ is an isomorphism. The following diagram illustrates this connection.

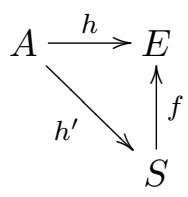

Now, suppose that we have $n$ evaluations expressed in a given ordinal scale $(S, \preccurlyeq)$ of cardinality $k=|S|$ and suppose we want to aggregate these evaluations and obtain a representative overall evaluation in the same ordinal scale. Of course, we can use a discrete aggregation function $G: S^{n} \rightarrow S$, which is nothing else than a ranking function sorting $k^{n}$ $n$-tuples into $k$ classes. (Here, "discrete" means that the domain of the function $G$ is a discrete set.) Alternatively, we can use a universal $n$ variable aggregation function that is independent of the ordinal scale used. In this latter case, since no scale can be specified, the aggregation function must be a numerical function $M: E^{n} \rightarrow E$. For instance, the classical median function, which gives the middle value of an oddlength sequence of ordered values, is a scale independent function able to aggregate numerical values expressed on any ordinal scale.

More exactly, we say that a function $M: E^{n} \rightarrow E$ is scale independent whenever it can be represented in any finite chain $(S, \preccurlyeq)$ by a symbolical analog $G: S^{n} \rightarrow S$ fulfilling

$$
M\left(x_{1}, \ldots, x_{n}\right)=f\left(G\left[f^{-1}\left(x_{1}\right), \ldots, f^{-1}\left(x_{n}\right)\right]\right) \quad\left(x \in E^{n}\right)
$$

for all isomorphism $f: S \rightarrow E$. As we will show, those functions are exactly the solutions of the functional equation

$$
M\left[\phi\left(x_{1}\right), \ldots, \phi\left(x_{n}\right)\right]=\phi\left[M\left(x_{1}, \ldots, x_{n}\right)\right] \quad\left(x \in E^{n}, \phi \in A(E)\right),
$$

which has been completely solved recently.

In this paper we define and investigate three types of scale independent functions for the aggregation on finite ordinal scales. First, we consider the functions mapping $n$ copies of the same ordinal scale into itself (see Definition 4.1), then the functions mapping $n$ copies of the same ordinal scale into an ordinal scale (see Definition 4.3), and finally the functions mapping $n$ independent ordinal scales into an ordinal scale (see Definition 4.5). We show that these functions identify with the so-called order invariant functions, which have already been investigated and completely described in a pure numerical setting [13] (see also $[1,12,15])$. Thus, our main contribution here is to interpret the order invariant functions as scale independent functions, that is, 
numerical functions that always have symbolical representatives when acting upon specified ordinal scales.

We also show that, even though at first glance it seems unappropriate to ask any scale independent function to be continuous, the continuity property can be interpreted in a very natural way for those scale independent functions of the first type.

The organization of the paper is as follows. In $\S 2$ we introduce the notation and the assumptions that we adopt in this work. In $\S 3$ we recall the concept of invariant subsets, which is necessary to describe the scale independent functions. In $\S 4$, we present separately the three types of scale independent functions mentioned above. Finally, in $\S 5$ we investigate the continuity property for those functions.

\section{Preliminaries and notation}

Let $E$ be any real interval, bounded or not. We denote by $B(E)$ the set of included boundaries of $E$, that is

$$
B(E):=\{\inf E, \sup E\} \cap E .
$$

The automorphism group of $E$, that is the group of all increasing bijections $\phi$ of $E$ onto itself, is denoted by $A(E)$. For the sake of simplicity, we also denote the index set $\{1, \ldots, n\}$ by $[n]$ and the minimum and maximum operations by $\wedge$ and $\vee$, respectively.

For any $k \geqslant 2$, a $k$-point ordinal scale $(S, \preccurlyeq)$ will be denoted by

$$
S=\left\{s_{1} \prec s_{2} \prec \cdots \prec s_{k}\right\}
$$

where $s_{*}=s_{1}$ (resp. $s^{*}=s_{k}$ ) is the bottom element (resp. top element) of the scale and $\prec$ represents the asymmetric part of $\preccurlyeq$.

As we have already mentioned in the introduction, the total order $\preccurlyeq$ defined on $S$ can always be represented in $E$ by means of an isomorphism $f: S \rightarrow E$, which is defined within an automorphism $\phi \in A(E)$. Throughout, we will assume that $f$ is endpoint preserving, that is, if $\inf E \in E($ resp. $\sup E \in E)$ then $f\left(s_{*}\right)=\inf E\left(\right.$ resp. $\left.f\left(s^{*}\right)=\sup E\right)$ for all ordinal scale $(S, \preccurlyeq)$. This amounts to assuming that the ordinal scales all have a common bottom element $s_{*}$ (resp. a common top element $s^{*}$ ) whose numerical representation is $\inf E(\operatorname{resp} . \sup E)$. This assumption clarifies why we consider numerical representations in a subset $E$ of $\mathbb{R}$, possibly nonopen, rather than $\mathbb{R}$ itself. For example, if $E=[0,1]$, all the ordinal scales we can consider have fixed endpoints.

To avoid a heavy notation, we will write $\phi(x)$ and $f(a)$ instead of

$$
\left(\phi\left(x_{1}\right), \ldots, \phi\left(x_{n}\right)\right) \text { and }\left(f\left(a_{1}\right), \ldots, f\left(a_{n}\right)\right),
$$


respectively. We will also write $\vec{\phi}(x)$ and $\vec{f}(a)$ instead of

$$
\left(\phi_{1}\left(x_{1}\right), \ldots, \phi_{n}\left(x_{n}\right)\right) \text { and }\left(f_{1}\left(a_{1}\right), \ldots, f_{n}\left(a_{n}\right)\right),
$$

respectively.

Finally, the range of any function $f$ will be denoted by $\operatorname{ran}(f)$.

\section{Background on invariant subsets}

In this section we recall the concept of invariant subset, which will be useful throughout this paper. For theoretical developments, see e.g. $[1,13,15]$.

DEFINITION 3.1. A nonempty subset $I \subseteq E^{n}$ is said to be invariant if

$$
x \in I \Rightarrow \phi(x) \in I \quad(\phi \in A(E)) .
$$

An invariant set I is said to be minimal if it has no proper invariant subset.

The family $\mathcal{I}\left(E^{n}\right)$ of all minimal invariant subsets of $E^{n}$, which consists exactly of the orbits of the elements of $E^{n}$ under the group

$$
\{(\underbrace{\phi, \ldots, \phi}_{n}) \mid \phi \in A(E)\}
$$

provides a partition of $E^{n}$ into equivalence classes, where $x, y \in E^{n}$ are equivalent if there exists $\phi \in A(E)$ such that $y=\phi(x)$. A complete description of elements of $\mathcal{I}\left(E^{n}\right)$ is given in the following proposition $[1,15]$.

PROPOSITION 3.1. We have $I \in \mathcal{I}\left(E^{n}\right)$ if and only if there exists a permutation $\pi$ on $[n]$ and a sequence $\left\{\triangleleft_{i}\right\}_{i=0}^{n}$ of symbols $\triangleleft_{i} \in\{<,=\}$, containing at least one symbol $<$ if inf $E \in E$ and $\sup E \in E$, such that

$$
I=\left\{x \in E^{n} \mid \inf E \triangleleft_{0} x_{\pi(1)} \triangleleft_{1} \cdots \triangleleft_{n-1} x_{\pi(n)} \triangleleft_{n} \sup E\right\},
$$

where $\triangleleft_{0}$ is $<$ if $\inf E \notin E$ and $\triangleleft_{n}$ is $<$ if $\sup E \notin E$.

EXAMPLE 3.1. The unit square $[0,1]^{2}$ contains exactly eleven minimal invariant subsets, namely the open triangles $\left\{\left(x_{1}, x_{2}\right) \mid 0<x_{1}<\right.$ $\left.x_{2}<1\right\}$ and $\left\{\left(x_{1}, x_{2}\right) \mid 0<x_{2}<x_{1}<1\right\}$, the open diagonal $\left\{\left(x_{1}, x_{2}\right) \mid\right.$ $\left.0<x_{1}=x_{2}<1\right\}$, the four square vertices, and the four open line segments joining neighboring vertices. 
Now we introduce another type of invariance.

DEFINITION 3.2. A nonempty subset $I \subseteq E^{n}$ is said to be strongly invariant if

$$
x \in I \Rightarrow \vec{\phi}(x) \in I \quad\left(\vec{\phi} \in A(E)^{n}\right) .
$$

A strongly invariant set $I$ is said to be minimal if it has no proper strongly invariant subset.

The family $\mathcal{I}^{*}\left(E^{n}\right)$ of all minimal strongly invariant subsets of $E^{n}$, which consists of the orbits of the elements of $E^{n}$ under the group $A(E)^{n}$, provides a partition of $E^{n}$ into equivalence classes, where $x, y \in$ $E^{n}$ are equivalent if there exists $\vec{\phi} \in A(E)^{n}$ such that $y=\vec{\phi}(x)$. A complete description of elements of $\mathcal{I}^{*}\left(E^{n}\right)$ is given in the following proposition [13].

PROPOSITION 3.2. We have

$$
\mathcal{I}^{*}\left(E^{n}\right)=\left\{\stackrel{n}{\times} I_{i=1} \mid I_{i} \in \mathcal{I}(E)\right\}=[\mathcal{I}(E)]^{n} .
$$

EXAMPLE 3.2. The unit square $[0,1]^{2}$ contains exactly nine minimal strongly invariant subsets, namely the open square $(0,1)^{2}$, the four square vertices, and the four open line segments joining neighboring vertices.

Observe that strong invariance is linked to invariance as follows. Two minimal invariant subsets $I$ and $J$ are said to be equivalent, $I \sim J$, if and only if for any $x \in I$ and any $u \in J$ there are $y, z \in I$ and $v, w \in J$ such that $y \leqslant u \leqslant z$ and $v \leqslant x \leqslant w$, where here $\leqslant$ represents the standard Cartesian partial order.

For any minimal invariant subset $I$, set

$$
I^{*}=\bigcup_{\substack{J \in \mathcal{I}\left(E^{n}\right) \\ J \sim I}} J
$$

Then (and only then) $I^{*}$ is a minimal strongly invariant subset; see [13].

\section{Scale independent functions}

In the present section we investigate the three kinds of scale independent functions we have mentioned in the introduction. Actually, we will 
show that these functions are nothing else than the so-called order invariant functions, namely: invariant functions, comparison meaningful functions, and strongly comparison meaningful functions.

\subsection{UNISCALE INDEPENDENT FUNCTIONS}

The first scale independent functions we investigate are $n$-variable numerical aggregation functions whose input and output values are expressed in the same ordinal scale. We call them uniscale independent functions.

DEFINITION 4.1. A function $M: E^{n} \rightarrow E$ is said to be uniscale independent if, for any finite ordinal scale $(S, \preccurlyeq)$, there exists an aggregation function $G: S^{n} \rightarrow S$ such that, for any endpoint preserving isomorphism $f: S \rightarrow E$, we have

$$
M[f(a)]=f[G(a)] \quad\left(a \in S^{n}\right) .
$$

We then say that $G$ represents $M$ in $(S, \preccurlyeq)$.

It is informative to represent Eq. (1) by the following commutative diagram

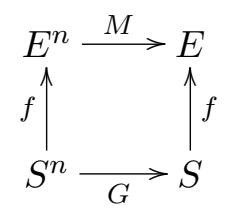

As any admissible scale transformation of the input values must lead to the same transformation of the output values, it seems that the uniscale independent functions are invariant functions in the following sense.

DEFINITION 4.2. $\quad M: E^{n} \rightarrow E$ is said to be an invariant function if

$$
M[\phi(x)]=\phi[M(x)]
$$

for all $x \in E^{n}$ and all $\phi \in A(E)$.

The invariant functions have been investigated extensively by several authors; see e.g. [12, 14, 15, 19]. Moreover, a full description of those functions has been given very recently as follows $[1,13,15]$.

THEOREM 4.1. $M: E^{n} \rightarrow E$ is an invariant function if and only if, for any $I \in \mathcal{I}\left(E^{n}\right)$ either $\left.M\right|_{I} \equiv c \in B(E)$ (if this constant exists) or there exists $i \in[n]$ such that $\left.M\right|_{I}=\left.P_{i}\right|_{I}$ is the projection onto the ith coordinate. 
Thus, an invariant function $M: E^{n} \rightarrow E$ reduces to a constant or a coordinate projection on every minimal invariant subset of $E^{n}$. In particular, we have

$$
M(x) \in\left\{x_{1}, \ldots, x_{n}\right\} \cup B(E) \quad\left(x \in E^{n}\right) .
$$

We now show that the uniscale independent functions coincide with the invariant functions.

PROPOSITION 4.1. The function $M: E^{n} \rightarrow E$ is uniscale independent if and only if it is invariant.

Proof. (Necessity) Let $M: E^{n} \rightarrow E$ be a uniscale independent function, let $x \in E^{n}$, let $\phi \in A(E)$, and let $(S, \preccurlyeq)$ be any finite ordinal scale with at least $n+|B(E)|$ elements. Then there exists $a \in S^{n}$ and an endpoint preserving isomorphism $f: S \rightarrow E$ such that $x=f(a)$. There exists also a function $G: S^{n} \rightarrow S$ such that

$$
M[f(a)]=f[G(a)]
$$

and

$$
\begin{aligned}
M[\phi(x)] & =M[(\phi \circ f)(a)]=(\phi \circ f)[G(a)]=\phi(M[f(a)]) \\
& =\phi[M(x)] .
\end{aligned}
$$

Hence $M$ is invariant.

(Sufficiency) Let $M: E^{n} \rightarrow E$ be an invariant function. Let $(S, \preccurlyeq)$ be any finite ordinal scale and let $f^{*}: S \rightarrow E$ be any fixed endpoint preserving isomorphism. Since $M$ satisfies (2), the function

$$
G(a):=f^{*-1}\left(M\left[f^{*}(a)\right]\right) \quad\left(a \in S^{n}\right)
$$

is well defined. Then, for any endpoint preserving isomorphism $f: S \rightarrow$ $E$, there exists $\phi \in A(E)$ such that $f=\phi \circ f^{*}$ and hence we have

$$
\begin{aligned}
M[f(a)] & =M\left[\left(\phi \circ f^{*}\right)(a)\right]=\phi\left(M\left[f^{*}(a)\right]\right)=\left(\phi \circ f^{*}\right)[G(a)] \\
& =f[G(a)]
\end{aligned}
$$

for all $a \in S^{n}$. Hence $M$ is uniscale independent.

According to Proposition 4.1, an invariant function $M: E^{n} \rightarrow E$ can always be represented by a discrete aggregation function $G: S^{n} \rightarrow S$ on any ordinal scale $(S, \preccurlyeq)$, regardless of the cardinality of this scale. Moreover, it is clear from Eq. (1) that $G$ is uniquely determined and, in some sense, it is isomorphic to the "restriction" of $M$ to $S^{n}$.

EXAMPLE 4.1. Let $n=2$ and let $M(x)=x_{1} \wedge x_{2}$. Then, the unique representative $G$ of $M$ is defined by $G(a)=a_{1} \wedge a_{2}$ for all $a \in S^{2}$. 
In fact, for a given ordinal scale $(S, \preccurlyeq)$, the set of functions $G: S^{n} \rightarrow$ $S$ representing invariant functions in $(S, \preccurlyeq)$ is described exactly as the discrete version of Theorem 4.1, where $E$ is replaced with $S$ and the family of "discrete" minimal invariant subsets of $S^{n}$ is simply defined either as

$$
\left\{f^{-1}(I) \mid I \in \mathcal{I}\left(E^{n}\right)\right\},
$$

for any fixed $f$, or independently of any $f$, by means of Proposition 3.1. Clearly, to have a one-to-one correspondence between $M$ and $G$ we need that $f^{-1}(I) \neq \varnothing$ for all $I \in \mathcal{I}\left(E^{n}\right)$, a condition that holds if and only if

$$
|S| \geqslant n+|B(E)|
$$

In this case, given $I \in \mathcal{I}\left(E^{n}\right)$ and $i \in[n]$, we have $\left.M\right|_{I}=\left.P_{i}\right|_{I}$ (resp. $\left.\left.M\right|_{I} \equiv \inf E,\left.M\right|_{I} \equiv \sup E\right)$ if and only if $G(a)=a_{i}\left(\right.$ resp. $G(a)=s_{*}$, $\left.G(a)=s^{*}\right)$ for all $a \in f^{-1}(I), f$ being fixed. On the other hand, if $|S|<n+|B(E)|$, several $M$ 's may lead to the same $G$. For example, if $n=2,|S|=3, E=[0,1]$, and $I \in \mathcal{I}\left([0,1]^{2}\right)$ is either of the two open triangles, then $f^{-1}(I)=\varnothing$ and, for a given $G$, the invariant function $M$ can take on any value in $I$.

Remark. That every invariant function $M: E^{n} \rightarrow E$ satisfies (2) is in accordance with the assumption that the input and output values are expressed in the same scale. Property (2) is also in agreement with the fact that, since no scale can be specified, the aggregated value must necessarily be one of the input values (or an endpoint of the scale if it is common to all the scales considered).

\subsection{INPUT-UNISCALE INDEPENDENT FUNCTIONS}

We now investigate scale independent functions whose input values are expressed in the same ordinal scale and the output values in an ordinal scale. We call these functions input-uniscale independent functions.

DEFINITION 4.3. A function $M: E^{n} \rightarrow \mathbb{R}$ is said to be input-uniscale independent if, for any finite ordinal scale $(S, \preccurlyeq S)$, there exists a finite ordinal scale $(T, \preccurlyeq T)$ and a surjective aggregation function $G: S^{n} \rightarrow T$ such that, for any endpoint preserving isomorphism $f: S \rightarrow E$, there is an isomorphism $g_{f}: T \rightarrow \mathbb{R}$ such that

$$
M[f(a)]=g_{f}[G(a)] \quad\left(a \in S^{n}\right) .
$$

We then say that $G$ represents $M$ in $(S, \preccurlyeq S)$. 
Eq. (3) can be graphically represented by the following commutative diagram

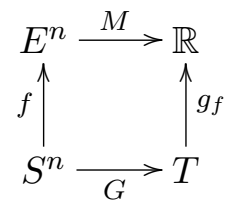

Just as we have shown that the uniscale independent functions are exactly the invariant functions, we will show in this subsection that the input-uniscale independent functions are exactly the comparison meaningful functions.

DEFINITION 4.4. $M: E^{n} \rightarrow \mathbb{R}$ is said to be a comparison meaningful function (from an ordinal scale) if

$$
M(x)\left\{\begin{array}{c}
< \\
=
\end{array}\right\} M\left(x^{\prime}\right) \Rightarrow M[\phi(x)]\left\{\begin{array}{c}
< \\
=
\end{array}\right\} M\left[\phi\left(x^{\prime}\right)\right]
$$

for any $x, x^{\prime} \in E^{n}$ and any $\phi \in A(E)$.

The comparison meaningful functions have been studied by various authors; see e.g. $[12,13,17,18,21]$. Moreover, the full description of those functions has been given very recently as follows [13].

THEOREM 4.2. $M: E^{n} \rightarrow \mathbb{R}$ is a comparison meaningful function if and only if, for any $I \in \mathcal{I}\left(E^{n}\right)$, there exists an index $i_{I} \in[n]$ and a constant or strictly monotonic function $g_{I}: P_{i_{I}}(I) \rightarrow \mathbb{R}$ such that

$$
\left.M\right|_{I}=\left.g_{I} \circ P_{i_{I}}\right|_{I},
$$

where, for any $I, J \in \mathcal{I}\left(E^{n}\right)$, either $g_{I}=g_{J}$, or $\operatorname{ran}\left(g_{I}\right)=\operatorname{ran}\left(g_{J}\right)$ is a singleton, or $\operatorname{ran}\left(g_{I}\right)<\operatorname{ran}\left(g_{J}\right)$, or $\operatorname{ran}\left(g_{I}\right)>\operatorname{ran}\left(g_{J}\right)$. (Note that $\operatorname{ran}\left(g_{I}\right)<\operatorname{ran}\left(g_{J}\right)$ means that for all $r \in \operatorname{ran}\left(g_{I}\right)$ and all $s \in \operatorname{ran}\left(g_{J}\right)$, we have $r<s$.)

Thus, a comparison meaningful function $M: E^{n} \rightarrow \mathbb{R}$ reduces to a constant or a transformed coordinate projection on every minimal invariant subset of $E^{n}$.

The following result clearly shows that comparison meaningfulness generalizes invariance.

PROPOSITION 4.2. $M: E^{n} \rightarrow \mathbb{R}$ is a comparison meaningful function if and only if, for any $\phi \in A(E)$, there is a strictly increasing mapping $\psi_{\phi}: \operatorname{ran}(M) \rightarrow \operatorname{ran}(M)$ such that

$$
M[\phi(x)]=\psi_{\phi}[M(x)] \quad\left(x \in E^{n}\right) .
$$


Proof. (Sufficiency) Trivial.

(Necessity) Let $\phi \in A(E)$ and assume that $M$ is comparison meaningful in $E^{n}$. For all $u \in \operatorname{ran}(M)$, there exists $x \in E^{n}$ such that $M(x)=u$. We then define $\psi_{\phi}(u)=M[\phi(x)]$. This function is strictly increasing since if $u, u^{\prime} \in \operatorname{ran}(M)$, there exist $x, x^{\prime} \in E^{n}$ such that $u=M(x)$ and $u^{\prime}=M\left(x^{\prime}\right)$, and hence

$$
\begin{aligned}
u<u^{\prime} & \Leftrightarrow M(x)<M\left(x^{\prime}\right) \\
& \Leftrightarrow M[\phi(x)]<M\left[\phi\left(x^{\prime}\right)\right] \\
& \Leftrightarrow \psi_{\phi}(u)<\psi_{\phi}\left(u^{\prime}\right)
\end{aligned}
$$

which completes the proof.

The previous result is in agreement with Definition 4.3 since any admissible transformation of the input values may lead to an admissible transformation of the output values. Moreover, it is clear from Eq. (4) that the function $\psi_{\phi}$ is uniquely determined.

We now show that the input-uniscale independent functions coincide with the comparison meaningful functions.

PROPOSITION 4.3. The function $M: E^{n} \rightarrow \mathbb{R}$ is input-uniscale independent if and only if it is comparison meaningful.

Proof. (Necessity) Let $M: E^{n} \rightarrow \mathbb{R}$ be an input-uniscale independent function, let $x \in E^{n}$, let $\phi \in A(E)$, and let $(S, \preccurlyeq S)$ be any finite ordinal scale with at least $n+|B(E)|$ elements. Then there exists $a \in S^{n}$ and an endpoint preserving isomorphism $f: S \rightarrow E$ such that $x=f(a)$. There exists also a finite ordinal scale $(T, \preccurlyeq T)$, a surjective aggregation function $G: S^{n} \rightarrow T$, and isomorphisms $g_{f}: T \rightarrow \mathbb{R}$ and $g_{\phi \circ f}: T \rightarrow \mathbb{R}$ such that

$$
M[f(a)]=g_{f}[G(a)]
$$

and

$$
M[(\phi \circ f)(a)]=g_{\phi \circ f}[G(a)] .
$$

Since there is an automorphism $\psi_{\phi}$ of $\mathbb{R}$ such that $g_{\phi \circ f}=\psi_{\phi} \circ g_{f}$, we have

$$
\begin{aligned}
M[\phi(x)] & =M[(\phi \circ f)(a)]=\left(\psi_{\phi} \circ g_{f}\right)[G(a)]=\psi_{\phi}(M[f(a)]) \\
& =\psi_{\phi}[M(x)] .
\end{aligned}
$$

It follows from Proposition 4.2 that $M$ is comparison meaningful.

(Sufficiency) Let $M: E^{n} \rightarrow \mathbb{R}$ be a comparison meaningful function. Let $(S, \preccurlyeq S)$ be any finite ordinal scale and let $f^{*}: S \rightarrow E$ be any fixed endpoint preserving isomorphism. Then the set

$$
R:=\left\{M\left[f^{*}(a)\right] \mid a \in S^{n}\right\}
$$


is a finite scale $\left\{r_{1}<\cdots<r_{|R|}\right\}$ in $\mathbb{R}$.

Define $T=\left\{t_{1} \prec \cdots \prec t_{|R|}\right\}$, then $\sigma: T \rightarrow R$, with $\sigma\left(t_{i}\right)=r_{i}$ for all $i=1, \ldots,|R|$, and finally $G: S^{n} \rightarrow T$ as

$$
G(a)=\sigma^{-1}\left(M\left[f^{*}(a)\right]\right) \quad\left(a \in S^{n}\right) .
$$

Then, $G$ is clearly surjective. Moreover, for any endpoint preserving isomorphism $f: S \rightarrow E$, there exists $\phi \in A(E)$ such that $f=\phi \circ f^{*}$ and, by Proposition 4.2, there exists a strictly increasing mapping $\psi_{\phi}$ : $\operatorname{ran}(M) \rightarrow \operatorname{ran}(M)$ such that

$$
\begin{aligned}
M[f(a)] & =M\left[\left(\phi \circ f^{*}\right)(a)\right]=\psi_{\phi}\left(M\left[f^{*}(a)\right]\right) \\
& =\left(\psi_{\phi} \circ \sigma\right)[G(a)]
\end{aligned}
$$

for all $a \in S^{n}$. Hence, it suffices to define $g_{f}: T \rightarrow \mathbb{R}$ as $g_{f}=\psi_{\phi} \circ \sigma$, where $\phi=f \circ f^{*-1}$, and $M$ is input-uniscale independent.

According to Proposition 4.3, a comparison meaningful function $M: E^{n} \rightarrow \mathbb{R}$ can always be represented by a discrete aggregation function $G: S^{n} \rightarrow T$ on any ordinal scale $(S, \preccurlyeq S)$, regardless of the cardinality of this scale. Moreover, the sufficiency part of the proof yields the necessary steps to determine the output scale $T$ and the functions $G: S^{n} \rightarrow T$ and $g_{f}: T \rightarrow \mathbb{R}$. These are:

Step 1. Fix a particular endpoint preserving isomorphism $f^{*}$ : $S \rightarrow E$.

Step 2. We have $T=\left\{t_{1} \prec \cdots \prec t_{|R|}\right\}$, where

$$
R:=\left\{M\left[f^{*}(a)\right] \mid a \in S^{n}\right\}=\left\{r_{1}<\cdots<r_{|R|}\right\} .
$$

Step 3. We have $G(a)=\sigma^{-1}\left(M\left[f^{*}(a)\right]\right)$, where $\sigma: T \rightarrow R$ is defined as $\sigma\left(t_{i}\right)=r_{i}$ for all $1 \leqslant i \leqslant|R|$.

Step 4. Determine the unique function $\psi_{\phi}: \operatorname{ran}(M) \rightarrow \operatorname{ran}(M)$ of Proposition 4.2.

Step 5. We have $g_{f}=\psi_{f \circ f^{*-1}} \circ \sigma$.

We clearly observe that, given $M: E^{n} \rightarrow \mathbb{R}$ and $(S, \preccurlyeq S)$, the scale $T$ and the functions $G: S^{n} \rightarrow T$ and $g_{f}: T \rightarrow \mathbb{R}$ are uniquely determined and do not depend upon the choice of $f^{*}$.

EXAMPLE 4.2. Let $M: E^{2} \rightarrow \mathbb{R}$ be defined by

$$
M(x)=g\left(x_{1} \wedge x_{2}\right)
$$


where $g: E \rightarrow \mathbb{R}$ is strictly decreasing. Then, given a $k$-point ordinal scale $(S, \preccurlyeq S)$ and an endpoint preserving isomorphism $f^{*}: S \rightarrow E$, we have

$$
R=\left\{g\left[f^{*}\left(s_{k}\right)\right]<\cdots<g\left[f^{*}\left(s_{1}\right)\right]\right\} .
$$

Then we have $|T|=k$ and the function $G: S^{n} \rightarrow T$ is given by

$$
G(a)=\left(\sigma^{-1} \circ g \circ f^{*}\right)\left(a_{1} \wedge a_{2}\right) \quad\left(a \in S^{2}\right),
$$

or equivalently by the following table

\begin{tabular}{c|cccc}
$a_{2} \backslash a_{1}$ & $s_{1}$ & $s_{2}$ & $\cdots$ & $s_{k}$ \\
\hline$s_{1}$ & $t_{k}$ & $t_{k}$ & $\cdots$ & $t_{k}$ \\
$s_{2}$ & $t_{k}$ & $t_{k-1}$ & $\cdots$ & $t_{k-1}$ \\
$\vdots$ & $\vdots$ & $\vdots$ & & $\vdots$ \\
$s_{k}$ & $t_{k}$ & $t_{k-1}$ & $\cdots$ & $t_{1}$
\end{tabular}

Finally, we have $\psi_{\phi}=g \circ \phi \circ g^{-1}$ and

$$
g_{f}\left(t_{i}\right)=\left(g \circ f \circ f^{*-1} \circ g^{-1} \circ \sigma\right)\left(t_{i}\right)=(g \circ f)\left(s_{k+1-i}\right)
$$

for $i=1, \ldots, k$.

EXAMPLE 4.3. Let $M:[0,1]^{2} \rightarrow \mathbb{R}$ be defined by

$$
M(x)=x_{1} \wedge x_{2}+2 \operatorname{sign}\left(x_{2}-x_{1}\right) .
$$

Then, given a 3-point ordinal scale $(S, \preccurlyeq S)$ and an endpoint preserving isomorphism $f^{*}: S \rightarrow E$, we have

$$
R=\{-2<z-2<0<z<1<2<z+2\},
$$

where $z=f^{*}\left(s_{2}\right)$. Then we have $|T|=7$ and the function $G: S^{n} \rightarrow T$ is given by

$$
G(a)=\sigma^{-1}\left[f^{*}\left(a_{1} \wedge a_{2}\right)+2 \operatorname{sign}\left(a_{2}-a_{1}\right)\right] \quad\left(a \in S^{2}\right),
$$

or equivalently by the following table

$$
\begin{array}{c|ccc}
a_{2} \backslash a_{1} & s_{1} & s_{2} & s_{3} \\
\hline s_{1} & t_{3} & t_{1} & t_{1} \\
s_{2} & t_{6} & t_{4} & t_{2} \\
s_{3} & t_{6} & t_{7} & t_{5}
\end{array}
$$

Finally, we have

$$
\psi_{\phi}(x)= \begin{cases}\phi(x), & \text { if } x \in[0,1], \\ \phi(x-2)+2, & \text { if } x \in[2,3), \\ \phi(x+2)-2, & \text { if } x \in[-2,-1) .\end{cases}
$$


and

$$
g_{f}\left(t_{i}\right)= \begin{cases}\left(f \circ f^{*-1}\right)\left[\sigma\left(t_{i}\right)\right], & \text { if } i=3,4,5, \\ \left(f \circ f^{*-1}\right)\left[\sigma\left(t_{i}\right)-2\right]+2, & \text { if } i=6,7, \\ \left(f \circ f^{*-1}\right)\left[\sigma\left(t_{i}\right)+2\right]-2, & \text { if } i=1,2 .\end{cases}
$$

Notice that the relationship between $M$ and $G$ is not as clear as in the case of uniscale independent functions. Particularly, reconstructing $M$ from $G$ (or characterizing $G$ arising from the $M$ 's) seems a difficult task. We then propose the following interesting problem.

Open Problem 1. Describe all the comparison meaningful functions having the same discrete representative.

Notice also that, from Eq. (3), we immediately have the following result, which will be very useful in the next section.

PROPOSITION 4.4. Let $M: E^{n} \rightarrow \mathbb{R}$ be an input-uniscale independent function, with discrete representative $G: S^{n} \rightarrow T$. Then, for any strictly increasing (resp. strictly decreasing) function $g: \operatorname{ran}(M) \rightarrow \mathbb{R}$, the discrete representative of $g \circ M$ is $\eta \circ G: S^{n} \rightarrow T^{\prime}$, where $T^{\prime}$ is order isomorphic to $T$ and $\eta: T \rightarrow T^{\prime}$ is defined by $\eta\left(t_{i}\right)=t_{i}^{\prime}$ (resp. $\left.\eta\left(t_{i}\right)=t_{|T|-i+1}^{\prime}\right)$ for all $i=1, \ldots,|T|$.

\subsection{INPUT-MULTISCALE INDEPENDENT FUNCTIONS}

The last functions we focus on are scale independent functions whose input values are expressed in independent ordinal scales and the output values in an ordinal scale. We call this third type of functions inputmultiscale independent functions.

DEFINITION 4.5. A function $M: E^{n} \rightarrow \mathbb{R}$ is said to be inputmultiscale independent if, for any finite ordinal scales $\left(S^{(i)}, \preccurlyeq S^{(i)}\right)(i \in$ $[n])$, there exists a finite ordinal scale $\left(T, \preccurlyeq_{T}\right)$ and a surjective aggregation function $G: \times_{i=1}^{n} S^{(i)} \rightarrow T$ such that, for any endpoint preserving isomorphisms $f_{i}: S^{(i)} \rightarrow E(i \in[n])$, there is an isomorphism $g_{\vec{f}}: T \rightarrow$ $\mathbb{R}$ such that

$$
M[\vec{f}(a)]=g_{\vec{f}}[G(a)] \quad\left(a \in \underset{i=1}{\times} S^{(i)}\right) .
$$

We then say that $G$ represents $M$ in $\times_{i=1}^{n}\left(S^{(i)}, \preccurlyeq S^{(i)}\right)$.

Here the commutative diagram is given by

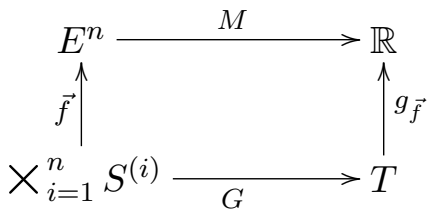


We will show in this subsection that the input-multiscale independent functions are exactly the strongly comparison meaningful functions.

DEFINITION 4.6. $\quad M: E^{n} \rightarrow \mathbb{R}$ is said to be a strongly comparison meaningful function (from independent ordinal scales) if

$$
M(x)\left\{\begin{array}{l}
< \\
=
\end{array}\right\} M\left(x^{\prime}\right) \quad \Rightarrow \quad M[\vec{\phi}(x)]\left\{\begin{array}{c}
< \\
=
\end{array}\right\} M\left[\vec{\phi}\left(x^{\prime}\right)\right]
$$

for any $x, x^{\prime} \in E^{n}$ and any $\vec{\phi} \in A(E)^{n}$.

The strongly comparison meaningful functions have been studied by some authors; see e.g. [8, 12, 13]. Moreover, the full description of those functions has been given as follows [13].

THEOREM 4.3. $M: E^{n} \rightarrow \mathbb{R}$ is a strongly comparison meaningful function if and only if, for any $I \in \mathcal{I}^{*}\left(E^{n}\right)$, there exists an index $i_{I} \in[n]$ and a constant or strictly monotonic function $g_{I}: P_{i_{I}}(I) \rightarrow \mathbb{R}$ such that

$$
\left.M\right|_{I}=\left.g_{I} \circ P_{i_{I}}\right|_{I},
$$

where, for any $I, J \in \mathcal{I}^{*}\left(E^{n}\right)$, either $g_{I}=g_{J}$, or $\operatorname{ran}\left(g_{I}\right)=\operatorname{ran}\left(g_{J}\right)$ is a singleton, or $\operatorname{ran}\left(g_{I}\right)<\operatorname{ran}\left(g_{J}\right)$, or $\operatorname{ran}\left(g_{I}\right)>\operatorname{ran}\left(g_{J}\right)$.

Thus, a strongly comparison meaningful function $M: E^{n} \rightarrow \mathbb{R}$ reduces to a constant or a transformed coordinate projection on every minimal strongly invariant subset of $E^{n}$.

PROPOSITION 4.5. $M: E^{n} \rightarrow \mathbb{R}$ is a strongly comparison meaningful function if and only if, for any $\vec{\phi} \in A(E)^{n}$, there is a strictly increasing mapping $\psi_{\vec{\phi}}: \operatorname{ran}(M) \rightarrow \operatorname{ran}(M)$ such that

$$
M[\vec{\phi}(x)]=\psi_{\vec{\phi}}[M(x)] \quad\left(x \in E^{n}\right) .
$$

Proof. The proof is almost identical to that of Proposition 4.2.

PROPOSITION 4.6. The function $M: E^{n} \rightarrow \mathbb{R}$ is input-multiscale independent if and only if it is strongly comparison meaningful.

Proof. The proof can be easily adapted from that of Proposition 4.3.

According to Proposition 4.6, a strongly comparison meaningful function $M: E^{n} \rightarrow \mathbb{R}$ can always be represented by a discrete aggregation function $G: \times_{i=1}^{n} S^{(i)} \rightarrow T$ on independent ordinal scales $\left(S^{(i)}, \preccurlyeq S_{(i)}\right)(i \in[n])$, regardless of the cardinalities of these scales. Moreover, the necessary steps to determine the output scale $T$ and the functions $G: \times_{i=1}^{n} S^{(i)} \rightarrow T$ and $g_{\vec{f}}: T \rightarrow \mathbb{R}$ are: 
Step 1. Fix a particular endpoint preserving isomorphism $f_{i}^{*}$ : $S^{(i)} \rightarrow E$ for all $i \in[n]$.

Step 2. We have $T=\left\{t_{1} \prec \cdots \prec t_{|R|}\right\}$, where

$$
R:=\left\{M\left[\overrightarrow{f^{*}}(a)\right] \mid a \in \times_{i=1}^{n} S^{(i)}\right\}=\left\{r_{1}<\cdots<r_{|R|}\right\} .
$$

Step 3. We have $G(a)=\sigma^{-1}\left(M\left[\vec{f}^{*}(a)\right]\right)$, where $\sigma: T \rightarrow R$ is defined as $\sigma\left(t_{i}\right)=r_{i}$ for all $1 \leqslant i \leqslant|R|$.

Step 4. Determine the unique function $\psi_{\vec{\phi}}: \operatorname{ran}(M) \rightarrow \operatorname{ran}(M)$ of Proposition 4.5.

Step 5. We have $g_{\vec{f}}=\psi_{\vec{f} \circ \vec{f}^{*-1}} \circ \sigma$.

EXAMPLE 4.4. Let $M: E^{2} \rightarrow \mathbb{R}$ be defined by $M(x)=g\left(x_{1}\right)$, where $g: E \rightarrow \mathbb{R}$ is strictly decreasing. Then, given $k_{i}$-point ordinal scales $\left(S^{(i)}, \preccurlyeq_{S^{(i)}}\right)(i \in[n])$ and endpoint preserving isomorphisms $f_{i}^{*}: S^{(i)} \rightarrow$ $E(i \in[n])$, we have

$$
R=\left\{g\left[f_{1}^{*}\left(s_{k_{1}}^{(1)}\right)\right]<\cdots<g\left[f_{1}^{*}\left(s_{1}^{(1)}\right)\right]\right\} .
$$

Then we have $|T|=k$ and $G(a)=\left(\sigma^{-1} \circ g \circ f_{1}^{*}\right)\left(a_{1}\right)$ for all $a \in$ $S^{(1)} \times S^{(2)}$. Finally, $\psi_{\vec{\phi}}=g \circ \phi_{1} \circ g^{-1}$ and

$$
g_{\vec{f}}\left(t_{i}\right)=\left(g \circ f_{1} \circ f_{1}^{*-1} \circ g^{-1} \circ \sigma\right)\left(t_{i}\right)=\left(g \circ f_{1}\right)\left(s_{k_{1}+1-i}\right)
$$

for $i=1, \ldots, k_{1}$.

\section{Continuous order invariant functions}

In this final section we examine the case of continuous order invariant functions, namely: continuous invariant functions, continuous comparison meaningful functions, and continuous strongly comparison meaningful functions.

One could think that coupling continuity with any order invariance property is somewhat awkward since the classical definition of continuity uses distance between numerical values and hence makes use of the cardinal properties of these values while any order invariance implies that the cardinal properties of the numerical values should not be used.

In fact, continuity is a topological concept which can be introduced for ordered sets and, as we will now show, it makes sense for invariant functions and can even be interpreted in a very natural way. In the first subsection, we yield an interpretation of continuity for invariant 
functions by imposing a smoothness property on their discrete representatives. In the second subsection, we provide another interpretation by imposing the admissible scale transformations to be nondecreasing (instead of being increasing bijections).

We also show that such interpretations fail to hold for comparison meaningful functions and strongly comparison meaningful functions and that continuity is a rather restrictive condition for those functions.

First, let us describe the continuous order invariant functions, a typical example of whose is given by a lattice polynomial [2].

DEFINITION 5.1. An n-variable lattice polynomial is any expression involving $n$ variables $x_{1}, \ldots, x_{n}$ linked by the lattice operations $\wedge=\min$ and $\vee=\max$ in an arbitrary combination of parentheses.

It can be shown (see e.g. [2, Chapter $2, \S 5]$ ) that any $n$-variable lattice polynomial in $\mathbb{R}^{n}$ can be put in the following disjunctive normal form

$$
L_{\gamma}(x)=\bigvee_{\substack{A \subseteq[n] \\ \gamma(A)=1}} \bigwedge_{i \in A} x_{i} \quad\left(x \in \mathbb{R}^{n}\right),
$$

where $\gamma: 2^{[n]} \rightarrow\{0,1\}$ is a nonconstant nondecreasing set function. We will denote by $\Gamma_{n}$ the family of those set functions.

The complete descriptions of continuous order invariant functions are given in the following three theorems $[12,13]$.

THEOREM 5.1. $M: E^{n} \rightarrow E$ is a continuous invariant function if and only if $M \equiv c \in B(E)$ (if this constant exists) or there exists $\gamma \in \Gamma_{n}$ such that $M=L_{\gamma}$.

THEOREM 5.2. $\quad M: E^{n} \rightarrow \mathbb{R}$ is a continuous comparison meaningful function if and only if there exists $\gamma \in \Gamma_{n}$ and a continuous strictly monotonic or constant function $g: E \rightarrow \mathbb{R}$ such that $M=g \circ L_{\gamma}$.

THEOREM 5.3. $\quad M: E^{n} \rightarrow \mathbb{R}$ is a continuous and strongly comparison meaningful function if and only if there exists $k \in N$ and a continuous strictly monotonic or constant function $g: E \rightarrow \mathbb{R}$ such that $M=g \circ P_{k}$.

\subsection{ORDER INVARIANT FUNCTIONS WITH SMOOTH DISCRETE REPRESENTATIVES}

We will now give an interpretation of the continuity property for invariant functions through their discrete representatives. For this purpose we use the concept of smoothness [5] for discrete functions. 
Let $(S, \preccurlyeq)=\left\{s_{1} \prec \cdots \prec s_{k}\right\}$ be a $k$-point ordinal scale and let $a \in S$. In order to locate $a$ in $S$ we define an index mapping

$$
\text { ind }: S \rightarrow\{1, \ldots, k\}
$$

as

$$
\operatorname{ind}(a)=r \quad \Leftrightarrow a=s_{r} \quad(1 \leqslant r \leqslant k) .
$$

DEFINITION 5.2. A discrete function $G: \times_{i=1}^{n} S^{(i)} \rightarrow T$ is said to be smooth if, for any $a, b \in \times_{i=1}^{n} S^{(i)}$, we have

$$
\sum_{i=1}^{n}\left|\operatorname{ind}\left(a_{i}\right)-\operatorname{ind}\left(b_{i}\right)\right| \leqslant 1 \Rightarrow|\operatorname{ind}[G(a)]-\operatorname{ind}[G(b)]| \leqslant 1 .
$$

The smoothness property, which was initially introduced only for nondecreasing discrete functions (see [5]), clearly represents the discrete counterpart of continuity. Moreover, it was proved in a particular case [4, Theorem 2] that this property is equivalent to the discrete counterpart of the intermediate value property. A complete proof of this equivalence is presented in the appendix.

We also have the following result that we will use in this subsection.

LEMMA 5.1. $G: \times_{i=1}^{n} S^{(i)} \rightarrow T$ is smooth if and only if

$$
|\operatorname{ind}[G(a)]-\operatorname{ind}[G(b)]| \leqslant \sum_{i=1}^{n}\left|\operatorname{ind}\left(a_{i}\right)-\operatorname{ind}\left(b_{i}\right)\right| \quad\left(a, b \in \underset{i=1}{\times} S^{(i)}\right) .
$$

Proof. (Necessity) Let $a, b \in \times_{i=1}^{n} S^{(i)}$ and set

$$
c^{(i)}:=\left(b_{1}, \ldots, b_{i}, a_{i+1} \ldots, a_{n}\right) \quad(i=0, \ldots, n),
$$

with $c^{(0)}=a$ and $c^{(n)}=b$. The result then follows from the immediate inequalities

$$
\left|\operatorname{ind}\left[G\left(c^{(i-1)}\right)\right]-\operatorname{ind}\left[G\left(c^{(i)}\right)\right]\right| \leqslant\left|\operatorname{ind}\left(a_{i}\right)-\operatorname{ind}\left(b_{i}\right)\right| \quad(i \in[n])
$$

and the classical Minkowski inequality

$$
|\operatorname{ind}[G(a)]-\operatorname{ind}[G(b)]| \leqslant \sum_{i=1}^{n}\left|\operatorname{ind}\left[G\left(c^{(i-1)}\right)\right]-\operatorname{ind}\left[G\left(c^{(i)}\right)\right]\right| .
$$

(Sufficiency) Trivial.

Observe that every lattice polynomial $M=L_{\gamma}$ in $E^{n}$, being a composition of $\vee, \wedge$, and coordinate projections, fulfills the so-called kernel property [9], namely

$$
|M(x)-M(y)| \leqslant \max _{i}\left|x_{i}-y_{i}\right| \quad\left(x, y \in E^{n}\right),
$$


which is much stronger than simple continuity. This kernel property can be naturally introduced also for discrete aggregation functions on ordinal scales as (see [16])

$$
|\operatorname{ind}[G(a)]-\operatorname{ind}[G(b)]| \leqslant \max _{i}\left|\operatorname{ind}\left(a_{i}\right)-\operatorname{ind}\left(b_{i}\right)\right| \quad\left(a, b \in \underset{i=1}{\times} S^{(i)}\right),
$$

which, according to Lemma 5.1, is a strengthening of the smoothness property. Evidently, each discrete representative $\left.L_{\gamma}\right|_{S^{n}}$ of a lattice polynomial $L_{\gamma}$ then necessarily satisfies this ordinal kernel property.

We now prove that any invariant function is continuous if and only if it is represented only by smooth discrete aggregation functions. This makes continuity sensible and even appealing for invariant functions. We will also show that this result does not hold for comparison meaningful functions and strongly comparison meaningful functions. More precisely, we will prove that continuity is only a sufficient condition for those functions to be represented only by smooth discrete functions.

PROPOSITION 5.1. An invariant function $M: E^{n} \rightarrow E$ is continuous if and only if it is represented only by smooth discrete aggregation functions.

Proof. (Necessity) Let $M: E^{n} \rightarrow E$ be a continuous invariant function. Then, by Theorem 5.1, either $M \equiv c \in B(E)$ or there exists $\gamma \in \Gamma_{n}$ such that $M=L_{\gamma}$. If $M \equiv c \in B(E)$ then the unique discrete representative $G: S^{n} \rightarrow S$ of $M$ in $(S, \preccurlyeq)$ is either $G \equiv s_{*}$ or $G \equiv s^{*}$, which is clearly smooth. Otherwise, if there exists $\gamma \in \Gamma_{n}$ such that $M=L_{\gamma}$, then the unique discrete representative $G: S^{n} \rightarrow S$ of $M$ in $(S, \preccurlyeq)$ is the "restriction" of $L_{\gamma}$ to $S^{n}$, which is smooth.

(Sufficiency) Suppose that $M$ is invariant but not continuous. We will show that there exists a finite ordinal scale $(S, \preccurlyeq)$ for which the unique discrete representative $G$ of $M$ in $(S, \preccurlyeq)$ is not smooth.

We can suppose without loss of generality that $E$ is bounded (if not, we apply any isomorphic transformation to some new bounded $E$ and all topological properties remain unchanged). Noncontinuity of $M$ means that there is $x \in E^{n}$ and a sequence $x^{(m)} \in E^{n}$ so that $x=\lim x^{(m)}, \lim M\left(x^{(m)}\right)=y$, but

$$
|M(x)-y|=3 \epsilon>0 .
$$

Fix $\delta>0$ such that $n \delta<\epsilon$. Then there exists an integer $m_{0}$ such that for $x^{*}=x^{\left(m_{0}\right)}$ the following holds

$$
\left|M(x)-M\left(x^{*}\right)\right|>2 \epsilon \quad \text { and } \quad\left|x_{i}-x_{i}^{*}\right|<\delta \quad \forall i \in[n] .
$$

Now, define

$$
Q:=\left\{M(x), M\left(x^{*}\right)\right\} \cup\left\{x_{i}, x_{i}^{*} \mid i \in[n]\right\},
$$




$$
\begin{aligned}
u & :=\min \left\{M(x), M\left(x^{*}\right)\right\}, \\
v & :=\max \left\{M(x), M\left(x^{*}\right)\right\} .
\end{aligned}
$$

Clearly $v-u=\left|M(x)-M\left(x^{*}\right)\right|>2 \epsilon$ and hence the Lebesgue measure of the set

$$
U:=(u, v) \backslash \bigcup_{i=1}^{n}\left(x_{i}-\delta, x_{i}+\delta\right)
$$

is greater than $2 \epsilon-2 n \delta>0$. Therefore, we can find $3 n^{2}$ different elements $z_{1}, \ldots, z_{3 n^{2}} \in U$. Then denote $Z:=\left\{z_{1}, \ldots, z_{3 n^{2}}\right\}$,

$$
P:=Q \cup Z \cup B(E)=\left\{p_{1}<\cdots<p_{|P|}\right\},
$$

and define an ordinal scale $S:=\left\{s_{1} \prec \cdots \prec s_{|P|}\right\}$. Introducing $\rho: S \rightarrow$ $P$, with $\rho\left(s_{i}\right)=p_{i}$ for all $1 \leqslant i \leqslant|P|$, we can define $G: S^{n} \rightarrow S$ as

$$
G(a)=\rho^{-1}(M[\rho(a)]) \quad\left(a \in S^{n}\right) .
$$

However, surely all elements of $Z$ are between $M(x)$ and $M\left(x^{*}\right)$, i.e.,

$$
\left|\operatorname{ind}\left(G\left[\rho^{-1}(x)\right]\right)-\operatorname{ind}\left(G\left[\rho^{-1}\left(x^{*}\right)\right]\right)\right|>3 n^{2} .
$$

On the other hand, for any $i \in[n]$, between $x_{i}$ and $x_{i}^{*}$ can be only elements from $Q$, so surely not more than $2 n$ elements from $P$, and thus

$$
\left|\operatorname{ind}\left[\rho^{-1}\left(x_{i}\right)\right]-\operatorname{ind}\left[\rho^{-1}\left(x_{i}^{*}\right)\right]\right| \leqslant 2 n+1 .
$$

However, then

$$
\sum_{i=1}^{n}\left|\operatorname{ind}\left[\rho^{-1}\left(x_{i}\right)\right]-\operatorname{ind}\left[\rho^{-1}\left(x_{i}^{*}\right)\right]\right| \leqslant n(2 n+1) \leqslant 3 n^{2} .
$$

Hence, by Lemma 5.1, $G$ is not smooth.

COROLLARY 5.1. An invariant function $M: E^{n} \rightarrow E$ is continuous if and only if it is represented only by kernel discrete aggregation functions.

Let us now examine the case of continuous comparison meaningful functions. By Proposition 4.4, we observe that any continuous invariant function of the form $L_{\gamma}$ and any nonconstant and continuous comparison meaningful function of the form $g \circ L_{\gamma}$, where $g$ is strictly increasing (resp. strictly decreasing), both lead to the representatives $L_{\gamma}: S^{n} \rightarrow S$ and $\eta \circ L_{\gamma}: S^{n} \rightarrow T$, respectively, where $T$ is order isomorphic to $S$, and $\eta: S \rightarrow T$ is the index-preserving (resp. index-reversing) mapping. This observation will be the key point to prove the remaining results of this subsection. 
PROPOSITION 5.2. A continuous comparison meaningful function $M: E^{n} \rightarrow \mathbb{R}$ is represented only by smooth discrete aggregation functions.

Proof. Let $M: E^{n} \rightarrow \mathbb{R}$ be a continuous comparison meaningful function. Then, by Theorem 5.2, there exists $\gamma \in \Gamma_{n}$ and a continuous strictly monotonic or constant function $g: E \rightarrow \mathbb{R}$ such that $M=g \circ L_{\gamma}$. If $g \equiv c \in \mathbb{R}$ then (see remark following Proposition 4.3) $G \equiv \sigma^{-1}(c) \equiv$ $t_{1}$ is a constant, hence a smooth function. Otherwise, according to the remark above, $G$ is necessarily of the form $\eta \circ L_{\gamma}$, where $\eta: S \rightarrow T$ is the index-preserving or index-reversing mapping. Hence $G$ is smooth.

COROLLARY 5.2. A continuous comparison meaningful function $M$ : $E^{n} \rightarrow \mathbb{R}$ is represented only by kernel discrete aggregation functions.

Back to Example 4.3, we can immediately see from the table describing the function $G$ that this function is not smooth. This is in accordance with the noncontinuity of $M$.

Notice that, contrary to the case of invariant functions, the converse of Proposition 5.2 is not true. There are noncontinuous comparison meaningful functions having smooth representatives. Indeed, starting from a strictly monotonic (but not necessarily continuous) $g: \mathbb{R} \rightarrow \mathbb{R}$, we can always transform a continuous invariant function $M: E^{n} \rightarrow$ $\mathbb{R}$ into the (not necessarily continuous) comparison meaningful function $g \circ M$, which has a smooth representative similar to that of $M$ (cf. Proposition 4.4). More precisely, for any strictly increasing (resp. strictly decreasing, constant) function $g: \mathbb{R} \rightarrow \mathbb{R}$, the unique representative in $(S, \preccurlyeq S)$ of $M=g \circ L_{\gamma}$ is the smooth function $G=\eta \circ L_{\gamma}$, where $\eta: S \rightarrow T=\operatorname{ran}(G)$ is index-preserving (resp. index-reversing, constant) and $|T|=|S|$ (resp. $|T|=|S|,|T|=1$ ).

The situation is similar for continuous strongly comparison meaningful functions, except that here the functions $L_{\gamma}$ reduce to coordinate projections.

PROPOSITION 5.3. A continuous strongly comparison meaningful function $M: E^{n} \rightarrow \mathbb{R}$ is represented only by smooth discrete aggregation functions.

COROLLARY 5.3. A continuous strongly comparison meaningful function $M: E^{n} \rightarrow \mathbb{R}$ is represented only by kernel discrete aggregation functions.

Thus, we can see that the continuity property is very restrictive for order invariant functions and imposes not only the smoothness property to the discrete representatives but also the kernel property, 
thus restricting the cardinality of the output scale $T$ to be not greater than the cardinality of the input scale $S$.

The following interesting problem naturally arises from this analysis.

Open Problem 2. Describe (or characterize) all the comparison meaningful functions and strongly comparison meaningful functions that are represented only by smooth discrete aggregation functions.

\subsection{ORder INVARIANT FUNCTIONS WITH NONDECREASING ADMISSIBLE TRANSFORMATIONS}

We now propose an alternative interpretation of the continuity property for invariant functions, which makes not use of discrete representatives.

Let $A^{\prime}(E)$ be the set of continuous nondecreasing surjections $\phi$ : $E \rightarrow E$. The following three results, inspired from [3, Proposition 2], show that the conjunction of order invariance and continuity is, in some sense, equivalent to requiring that the admissible scale transformations belong to $A^{\prime}(E)$.

PROPOSITION 5.4. $\quad M: E^{n} \rightarrow E$ is a continuous invariant function if and only if

$$
M[\phi(x)]=\phi[M(x)]
$$

for all $x \in E^{n}$ and all $\phi \in A^{\prime}(E)$.

Proof. (Necessity) The result is immediate if $M \equiv c \in B(E)$. Otherwise, by Theorem 5.1, there exists $\gamma \in \Gamma_{n}$ such that $M=L_{\gamma}$. Then, for any $x \in E^{n}$ and any $\phi \in A^{\prime}(E)$, we have

$$
M[\phi(x)]=L_{\gamma}[\phi(x)]=\phi\left[L_{\gamma}(x)\right]=\phi[M(x)] .
$$

(Sufficiency) Clearly, $M$ is invariant. Suppose that it is not continuous. Then there exist distinct minimal invariant subsets $I, J \in$ $\mathcal{I}\left(E^{n}\right)$ and a sequence $z^{(m)} \in I$ converging to an element $z \in J$ such that $M\left(z^{(m)}\right)$ does not converge to $M(z)$. Because of Theorem 4.1, this means that $\lim M\left(z^{(m)}\right)=z_{i}$ and $M(z)=z_{j}$ for some $i, j \in$ $\{0,1, \ldots, n, n+1\}$ so that $z_{i} \neq z_{j}$, with the convention that $z_{0}=\inf E$ and $z_{n+1}=\sup E$ if $B(E) \neq \varnothing$.

Denote by $k_{1}, k_{2}, \ldots, k_{l}(l \leqslant n)$ the distinct values of $\left\{z_{1}, \ldots, z_{n}\right\}$. Suppose without loss of generality that

$$
\inf E=k_{0} \leqslant k_{1}<k_{2}<\cdots<k_{l} \leqslant k_{l+1}=\sup E
$$

and define

$$
\delta=\min _{\substack{j=1, \ldots, l+1 \\ k_{j}-k_{j-1}>0}}\left(k_{j}-k_{j-1}\right) .
$$


Since $z^{(m)}$ converges to $z$, we can find a natural integer $p$ such that

$$
\left|z_{i}^{(p)}-z_{i}\right|<\delta / 4 \quad(i \in[n])
$$

Now, define a continuous nondecreasing surjection $\phi: E \rightarrow E$ such that, for any $j \in\{1, \ldots, l\}$, we have $\phi(x)=k_{j}$ whenever $\left|x-k_{j}\right| \leqslant \delta / 3$.

It follows that $\phi \in A^{\prime}(E)$ and $\phi\left(z^{(p)}\right)=z$. Finally, we have

$$
z_{i}=\phi\left(z_{i}^{(p)}\right)=\phi\left[M\left(z^{(p)}\right)\right]=M\left[\phi\left(z^{(p)}\right)\right]=M(z)=z_{j},
$$

a contradiction.

PROPOSITION 5.5. Let $M: E^{n} \rightarrow \mathbb{R}$ be any function. Consider the following four assertions:

i) $M$ is a continuous comparison meaningful function.

ii) For any $\phi \in A^{\prime}(E)$, there is a continuous and nondecreasing mapping $\psi_{\phi}: \operatorname{ran}(M) \rightarrow \operatorname{ran}(M)$ such that

$$
M[\phi(x)]=\psi_{\phi}[M(x)] \quad\left(x \in E^{n}\right) .
$$

iii) For any $\phi \in A^{\prime}(E)$, there is a nondecreasing mapping $\psi_{\phi}: \operatorname{ran}(M) \rightarrow$ $\operatorname{ran}(M)$ such that

$$
M[\phi(x)]=\psi_{\phi}[M(x)] \quad\left(x \in E^{n}\right) .
$$

iv) We have

$$
M(x)\left\{\begin{array}{l}
< \\
=
\end{array}\right\} M\left(x^{\prime}\right) \Rightarrow M[\phi(x)]\left\{\begin{array}{l}
\leqslant \\
=
\end{array}\right\} M\left[\phi\left(x^{\prime}\right)\right]
$$

for any $x, x^{\prime} \in E^{n}$ and any $\phi \in A^{\prime}(E)$.

Then we have $i) \Leftrightarrow i i), i i) \Rightarrow i i i), i i i) \Leftrightarrow i v)$, and $i v) \nRightarrow i)$.

Proof. $i) \Rightarrow i i)$ The result is immediate if $M$ is a constant function. Otherwise, by Theorem 5.2, there exists $\gamma \in \Gamma_{n}$ and a continuous strictly monotonic function $g: E \rightarrow \mathbb{R}$ such that $M=g \circ L_{\gamma}$. Then, for any $\phi \in A^{\prime}(E)$, we have

$$
M[\phi(x)]=\left(g \circ L_{\gamma}\right)[\phi(x)]=(g \circ \phi)\left[L_{\gamma}(x)\right]=\psi_{\phi}[M(x)] \quad\left(x \in E^{n}\right),
$$

where $\psi_{\phi}=g \circ \phi \circ g^{-1}$ is a continuous and nondecreasing mapping.

$i i) \Rightarrow i$ ) We can easily see that $M$ is comparison meaningful. Suppose that it is not continuous. Then there exists minimal invariant subsets $I, J \in \mathcal{I}\left(E^{n}\right)$ and a sequence $z^{(m)} \in I$ converging to an element 
$z \in J$ such that $M\left(z^{(m)}\right)$ does not converge to $M(z)$. Contrary to the sufficiency part of Proposition 5.4, we do not assume here that $I$ and $J$ are distinct.

Let us first show that we can require without loss of generality that $z^{(m)}$ is a nondecreasing or nonincreasing (in each coordinate) sequence.

By Theorem 4.2, $M\left(z^{(m)}\right)$ is of the form

$$
M\left(z^{(m)}\right)=g_{I}\left(z_{i_{I}}^{(m)}\right) .
$$

Since the real sequence $z_{i_{I}}^{(m)}$ converges to $z_{i_{I}}$, we can always choose a monotone subsequence $z_{i_{I}}^{\left(k_{m}\right)}$ converging to the same limit.

From the structure of minimal invariant subsets it follows that there always exists a nondecreasing or nonincreasing sequence $d^{(m)} \in I$ converging to $z \in J$. Choose $d^{(m)}$ to be nondecreasing or nonincreasing depending on whether $z_{i_{I}}^{\left(k_{m}\right)}$ is nondecreasing or nonincreasing. Define the sequence $c^{(m)} \in I$ by

$$
c_{i}^{(m)}= \begin{cases}z_{i_{I}}^{\left(k_{m}\right)}, & \text { if } i=i_{I}, \\ d_{i}^{(m)}, & \text { otherwise. }\end{cases}
$$

Clearly, $c^{(m)}$ converges to $z \in J$ and $M\left(c^{(m)}\right)=M\left(z^{\left(k_{m}\right)}\right)$ does not converge to $M(z)$.

Therefore, replacing $z^{(m)}$ with $c^{(m)}$ if necessary, we can assume that $z^{(m)}$ is monotone. Let us assume that it is nondecreasing. The other case can be dealt with similarly.

Consider now the continuous surjection $\phi: E \rightarrow E$ constructed in the proof of Proposition 5.4 and define its pseudo-inverse

$$
\phi^{(-1)}(t):=\sup \{x \in E \mid \phi(x)<t\},
$$

which is nondecreasing and left-continuous, and define

$$
y^{(m)}:=\phi^{(-1)}\left(z^{(m)}\right) .
$$

Then, we have $y:=\lim y^{(m)}=\phi^{(-1)}(z)$ and

$$
M\left(z^{(m)}\right)=M\left[\phi\left(y^{(m)}\right)\right]=\psi_{\phi}\left[M\left(y^{(m)}\right)\right]
$$

is a converging sequence whose limit is different from

$$
M(z)=M[\phi(y)]=\psi_{\phi}[M(y)] .
$$

Since $\psi_{\phi}$ is continuous, $M$ must be noncontinuous in $y$. Moreover, since $z^{(m)}$ is nondecreasing, we have $y_{i}=z_{i}-\delta / 3$ for all $i \in[n]$ and hence 
$y \in J$. However, exactly the same procedure can be repeated with $\delta / \alpha$ for any $\alpha \in[3,+\infty)$, which implies that all the points of the line segment joining $y$ to $z$ are discontinuities of $M$. If $I=J$, this means that $g_{I}$ is a strictly monotone function having uncountably many discontinuities, which is impossible. If $I \neq J$ and $g_{I}=g_{J}$, again $g_{I}$ has uncountably many discontinuities, which is impossible. Finally, let us consider the case where $\operatorname{ran}\left(g_{I}\right)$ and $\operatorname{ran}\left(g_{J}\right)$ are disjoint. Clearly, there always exists $\phi \in A^{\prime}(E)$ such that $\phi\left(z^{(m)}\right) \in J$ for some values of $m$ and $\phi\left(z^{(m)}\right) \in I$ for the others. Then we have

$$
M\left[\phi\left(z^{(m)}\right)\right]=\psi_{\phi}\left[M\left(z^{(m)}\right)\right]=\psi_{\phi}\left[g_{I}\left(z_{i_{I}}^{(m)}\right)\right],
$$

that is

$$
\psi_{\phi}\left[g_{I}\left(z_{i_{I}}^{(m)}\right)\right] \in \begin{cases}\operatorname{ran}\left(g_{J}\right), & \text { if } \phi\left(z^{(m)}\right) \in J \\ \operatorname{ran}\left(g_{I}\right), & \text { if } \phi\left(z^{(m)}\right) \in I .\end{cases}
$$

Since $\psi_{\phi}$ is continuous, we get a contradiction.

$i i) \Rightarrow$ iii) Trivial.

iii) $\Leftrightarrow i v$ ) Similar to the proof of Proposition 4.2.

$i v) \nRightarrow i$ ) A counter-example can be given by any discontinuous and strictly increasing one-variable function $M: E \rightarrow \mathbb{R}$.

PROPOSITION 5.6. Let $M: E^{n} \rightarrow \mathbb{R}$ be any function. Consider the following four assertions:

i) $M$ is a continuous strongly comparison meaningful function.

ii) For any $\vec{\phi} \in A^{\prime}(E)^{n}$, there is a continuous and nondecreasing mapping $\psi_{\vec{\phi}}: \operatorname{ran}(M) \rightarrow \operatorname{ran}(M)$ such that

$$
M[\vec{\phi}(x)]=\psi_{\vec{\phi}}[M(x)] \quad\left(x \in E^{n}\right) .
$$

iii) For any $\vec{\phi} \in A^{\prime}(E)^{n}$, there is a nondecreasing mapping $\psi_{\vec{\phi}}$ : $\operatorname{ran}(M) \rightarrow \operatorname{ran}(M)$ such that

$$
M[\vec{\phi}(x)]=\psi_{\vec{\phi}}[M(x)] \quad\left(x \in E^{n}\right) .
$$

iv) We have

$$
M(x)\left\{\begin{array}{l}
< \\
=
\end{array}\right\} M\left(x^{\prime}\right) \quad \Rightarrow \quad M[\vec{\phi}(x)]\left\{\begin{array}{c}
\leqslant \\
=
\end{array}\right\} M\left[\vec{\phi}\left(x^{\prime}\right)\right]
$$

for any $x, x^{\prime} \in E^{n}$ and any $\vec{\phi} \in A^{\prime}(E)^{n}$.

Then we have $i) \Leftrightarrow i i), i i) \Rightarrow i i i),(i i) \Leftrightarrow i v)$, and $i v) \nRightarrow i)$. 
Proof. Similar to the proof of Proposition 5.5.

\section{Concluding remarks}

We have shed light on the meaning of invariant functions by interpreting them as scale independent functions, that is, functions that have discrete representatives on any finite ordinal scale.

In particular, this interpretation shows that considering a discrete function $G: S^{n} \rightarrow S$, where $(S, \preccurlyeq)$ is a given ordinal scale, is not equivalent to considering an invariant function $M: E^{n} \rightarrow E$. Indeed, the latter form is much more restrictive since $M$ is independent of any scale. For instance, if $n=2$ and $E$ is open, we see by Theorem 4.1 that there are only 4 invariant functions (since $E^{2}$ has only three minimal invariant subsets and there is only one possibility on the diagonal) while the number of discrete functions $G: S^{2} \rightarrow S$ is clearly $|S|^{|S|^{2}}$.

We have also interpreted the comparison meaningful functions and the strongly comparison meaningful functions in a similar way. In this case, describing all the order invariant functions leading to the same discrete representative remains an interesting open problem.

Finally, we have observed that these interpretations make the continuity property very sensible for invariant functions and, however, rather restrictive for comparison meaningful functions and strongly comparison meaningful functions.

We believe that such interpretations can also be made on aggregation functions acting on other scale types, such as nominal scales.

\section{Appendix}

\section{A. Discrete intermediate value theorem}

Fodor [4, Theorem 2] showed that, for a nondecreasing discrete function $G: S^{2} \rightarrow S$, the smoothness condition is equivalent to a discrete analog of the intermediate value property. Here we state and prove this result in the general case.

PROPOSITION A.1. The smoothness property for $G: \times_{i=1}^{n} S^{(i)} \rightarrow$ $T$ is equivalent to the following condition: For any $j \in[n]$ and any $a, b \in \times_{i=1}^{n} S^{(i)}$ differing only on coordinate $j$, the element $t \in T$ lies between $G(a)$ and $G(b)$ inclusive if and only if there exists $c \in$ $\times_{i=1}^{n} S^{(i)}$, differing from $a$ and $b$ only on coordinate $j$, such that $c_{j}$ is an element between $a_{j}$ and $b_{j}$ inclusive and $t=G(c)$. 
Proof. (Necessity) Let $G: \times_{i=1}^{n} S^{(i)} \rightarrow T$ be a smooth function and let $a, b \in \times_{i=1}^{n} S^{(i)}$ differ only on coordinate $j$. We can assume without loss of generality that $a_{j}=s_{p}$ and $b_{j}=s_{q}$, with $p \leqslant q \leqslant\left|S^{(j)}\right|$. For any integer $0 \leqslant m \leqslant q-p$, we define $c^{(m)} \in \mathbf{X}_{i=1}^{n} S^{(i)}$ differing from $a$ and $b$ only on coordinate $j$ and such that $c_{j}^{(m)}=s_{p+m}$. Since $G$ is smooth, we have

$$
\left|\operatorname{ind}\left[G\left(c^{(m)}\right)\right]-\operatorname{ind}\left[G\left(c^{(m-1)}\right)\right]\right| \leqslant 1
$$

for all $1 \leqslant m \leqslant q-p$. Hence, for any $t$ lying between $G(a)$ and $G(b)$, there exists $0 \leqslant m \leqslant q-p$ such that $t=G\left(c^{(m)}\right)$. Conversely, it is clear that any $G\left(c^{(m)}\right)(0 \leqslant m \leqslant q-p)$ lies between $G(a)$ and $G(b)$.

(Sufficiency) Suppose $G: \times_{i=1}^{n} S^{(i)} \rightarrow T$ is not smooth and however fulfills the discrete intermediate value theorem. Then, there exists $a \in$ $\times_{i=1}^{n} S^{(i)}, j \in[n], 2 \leqslant r \leqslant\left|S^{(j)}\right|$, and $1 \leqslant p, q \leqslant|T|$ such that

$$
\begin{aligned}
t_{p} & =G\left(a \mid a_{j}=s_{r}\right) \\
t_{q} & =G\left(a \mid a_{j}=s_{r-1}\right)
\end{aligned}
$$

where $q<p-1$ or $q>p+1$. We can assume without loss of generality that $q<p-1$. Then we have

$$
G\left(a \mid a_{j}=s_{r-1}\right)=t_{q} \prec t_{p-1} \prec t_{p}=G\left(a \mid a_{j}=s_{r}\right)
$$

and there exists $c \in S^{(j)}$ fulfilling $s_{r-1} \preccurlyeq c \preccurlyeq s_{r}$ such that

$$
t_{p-1}=G\left(a \mid a_{j}=c\right) \text {. }
$$

Hence, $c=s_{r}$ or $c=s_{r-1}$, a contradiction.

\section{References}

1. L. Bartłomiejczyk and J. Drewniak, A characterization of sets and operations invariant under bijections, Aequationes Math. 68 (1) (2004) 1-9.

2. G. Birkhoff, Lattice Theory, (Third Edition, AMS, Providence, 1967).

3. D. Bouyssou and M. Pirlot, Choosing and ranking on the basis of fuzzy preference relations with the "min in favor". Multiple criteria decision making (Hagen, 1995), Lecture Notes in Econom. and Math. Systems, Vol. 448 (Springer, Berlin, 1997) 115-127.

4. J. Fodor, Smooth associative operations on finite ordinal scales, IEEE Trans. Fuzzy Syst. 8 (6) (2000) 791-795.

5. L. Godó and C. Sierra, A new approach to connective generation in the framework of expert systems using fuzzy logic, Proc. 18th IEEE Int. Symposium on Multiple-Valued Logic, Palma de Mallorca, Spain, 1988, pp. 157-162. 
6. M. Grabisch, On preference representation on an ordinal scale, in: S. Benferhat, P. Besnard (eds.), Proc. 6th Eur. Conf. on Symbolic and Quantitative Approaches to Reasoning with Uncertainty (ECSQARU 2001), Toulouse, France, September 19-21, 2001, pp. 18-28.

7. M. Grabisch and T. Roblin, Aggregation of ordinal information, Proc. of EUROFUSE-SIC'99 Joint Conference, Budapest, Hungary, May 25-28, 1999, pp. 496-501.

8. S.-R. Kim, On the possible scientific laws, Math. Social Sci. 20 (1) (1990) 19-36.

9. A. Kolesárová and J. Mordelová, 1-Lipschitz and kernel aggregation operators, Proc. Int. Summer School on Aggregation Operators and their Applications (AGOP 2001), Asturias, Spain, July 10-13, 2001, pp. 71-75.

10. D.H. Krantz, R.D. Luce, P. Suppes, and A. Tversky, Foundations of measurement, volume I: Additive and polynomial representations (Academic Press, San Diego, 1971).

11. R.D. Luce, D.H. Krantz, P. Suppes, and A. Tversky, Foundations of measurement, volume III: Representation, axiomatization, and invariance (Academic Press, San Diego, 1990).

12. J.-L. Marichal, On order invariant synthesizing functions, J. of Math. Psych. 46 (6) (2002) 661-676.

13. J.-L. Marichal, R. Mesiar, and T. Rückschlossová, A complete description of comparison meaningful functions, Aequationes Math., in press.

14. J.-L. Marichal and M. Roubens, Characterization of some stable aggregation functions, Proc. 1st Int. Conf. on Industrial Engineering and Production Management (IEPM'93), Mons, Belgium, June 2-4, 1993, pp. 187-196.

15. R. Mesiar and T. Rückschlossová, Characterization of invariant aggregation operators, Fuzzy Sets and Systems 142 (1) (2004) 63-73.

16. J. Mordelová and E. Muel, Binary jointly strictly monotone kernel aggregation operators on finite scales, Proc. Uncertainty 2001, Bratislava, Slovakia, September 2001, pp. 127-131.

17. A. Orlov, The connection between mean quantities and admissible transformations, Math. Notes 30 (1981) 774-778.

18. S. Ovchinnikov, Means on ordered sets, Math. Social Sci. 32 (1) (1996) 39-56.

19. S. Ovchinnikov, Invariant functions on simple orders, Order 14 (4) (1998) 365371

20. F.S. Roberts, Measurement theory (with applications to decision-making, utility and the social sciences), (Addison-Wesley Pub., Reading, MA, 1979).

21. E. Yanovskaya, Group choice rules in problems with interpersonal preference comparisons, Automat. Remote Control 50 (6) (1989) 822-830. 